\title{
La pragmaticalisation de l'adverbe enfin du point de vue des romanistes (« Enfin, de celui des francisants qui conçoivent leur recherche dans le cadre de la linguistique romane ")
}

\author{
Éva Buchi, \\ ATILF (Nancy-Université \& CNRS) \\ eva.buchi@atilf.fr \\ Thomas Städtler \\ DEAF (Académie des Sciences de Heidelberg) \\ thomas.staedtler@urz.uni-heidelberg.de
}

\section{Introduction}

\subsection{Contexte de recherche}

Cette communication ${ }^{1}$ se situe dans le cadre théorique des «mots du discours en tant que mots de la langue » (Paillard, 1998) et de la (poly-)pragmaticalisation (Dostie, 2004). Visant, dans la continuité des travaux de l'une d'entre nous (Buchi, 2007a ; 2007b ; à paraître), à mettre en évidence l'échelonnement diachronique des différentes valeurs, tant grammaticales que pragmatiques, que connaît l'adverbe enfin en français contemporain, elle fait le pari d'un va-et-vient fructueux entre analyse linguistique et analyse philologique ${ }^{2}$.

\subsection{Historique des études consacrées à enfin}

Le dernier quart de siècle a vu paraitre pas moins de dix-sept travaux entièrement ou partiellement consacrés à une description synchronique du marqueur enfin du français contemporain (Cadiot et al., 1985 ; Oguma, 1987 ; Franckel, 1989 ; Luscher \& Moeschler, 1990 ; Hwang, 1993 ; Barnes, 1995 ; Rossari, 1997 et 2000 ; Nemo, 2000 ; Beeching, 2001, 2002 et 2007 ; Paillard, 2003 ; Bertrand \& Chanet, 2005 ; Hansen, 2005b), y compris deux études contrastives (avec l'allemand: Métrich, 1985; avec le danois : Nølke, 1991). À l'opposé de cette prolifération de publications d'inspiration synchronique, l'approche diachronique n'a guère passionné les chercheurs: seule M.-B. Hansen (2005a [cf. aussi 2005b]) a pris à bras-le-corps, dans le cadre d'une étude d'inspiration cognitive-fonctionnelle, la question de la genèse des différentes valeurs de enfin.

\subsection{Objectif et méthode}

Conscients du déséquilibre patent entre publications synchroniques et diachroniques consacrées à enfin, nous nous proposons de compléter l'étude pionnière de M.-B. Hansen (2005a) par une approche qui se distingue de la sienne à double titre. D'une part, là où elle s'appuyait sur un corpus exclusivement constitué de bases de données, notre analyse se fondera sur la totalité des sources lexicographiques et électroniques disponibles, y compris la documentation inédite du DEAF. D'autre part, étant donné qu'une des hypothèses sous-jacentes de nos recherches respectives consiste à poser que la linguistique historique française se conçoit avec profit comme une branche de la linguistique romane, nous aurons recours au témoignage de l'ensemble des langues romanes pour reconstruire la protohistoire du français. 


\section{Analyse}

Des correspondants (tant formels que sémantiques) du grammème français enfin se relèvent dans sept autres langues romanes : sarde (campidanais) in finis (où $-s$ représente une marque adverbiale, DES s.v. fine $^{1}$ ), italien infine (dp. 1240/1250 ; OVI ; DELI ${ }^{2}$; GDLI), frioulan infin (dp. $16^{\mathrm{e}}$ s., Joppi $1878: 241$; Pirona, 1992), occitan enfin (dp. 1460, FEW 3, 561a), gascon enfí (Palay), espagnol enfin (1344-1787; CORDE ; NTLLE) et portugais enfim (dp. $15^{\mathrm{e}}$ s. ; Cunha, 2002 ; Houaiss ; DLPC ; Ø DELP ${ }^{3}$; Ø DENF) ${ }^{3}$. A priori, cette situation supporte quatre explications : il s'agirait d'un universel linguistique ; de l'héritage d'un ancêtre commun, le protoroman (ou, si l'on préfère, le latin vulgaire); de l'effet d'une série d'emprunts intra-romans ; enfin d'une convergence fortuite. L'hypothèse d'un universel peut être écartée aisément : si certaines langues non romanes connaissent des adverbes signifiant « enfin » construits sur des lexèmes signifiant «fin » (ainsi all. endlich ou russ. наконец), elles ne font pas toutes intervenir l'équivalent de la préposition en. Pour ce qui est de l'hypothèse de développements indépendants à l'époque romane, elle se heurte à une difficulté majeure : la probabilité que huit idiomes apparentés en viennent à créer de façon indépendante le même grammème est bien faible. Quant à l'hypothèse d'une série d'emprunts à partir d'un seul idiome roman, elle n'est supportée par aucun argument (l'emprunt grammatical présuppose d'ailleurs une situation sociolinguistique bien particulière). Nous défendons donc, sauf cas particuliers éventuels, l'hypothèse de l'héritage commun : fr. enfin représente, comme ses congénères sarde, italien, frioulan, occitan, gascon, espagnol et portugais, le continuateur de protoroman */In'fine/ adv. « à la fin ».

On relève le corrélat infine de ce grammème reconstruit du protoroman en latin écrit de l'Antiquité $\left(1^{\mathrm{er}} \mathrm{s}\right.$. av. J.-Chr. $-4^{\mathrm{e}} / 5^{\mathrm{e}}$ s. [saint Augustin] ; OLD ; Reichenberger, $1956: 148$, n.2 ; Bambeck, 1958 : 128), ce qui a incité $\mathrm{DELI}^{2}$ à postuler, à juste titre, le caractère héréditaire d'it. infine (« lat. tardo [S. Agostino] in fine »). À cette exception notable près, les ouvrages de référence analysent toutefois fr. enfin et ses congénères - à tort, si l'on accepte notre raisonnement - comme des formations romanes ${ }^{4}$.

\subsection{Grammème}

\subsubsection{Enfin1 temporel}

Un premier sens de enfin pourrait être rendu par «à la fin, pour terminer (indique qu'un procès est le dernier d'une série) » (TLF 1979 [I.A.] ; Cadiot et al., 1985 : 203-205 [2.A.1.a.] ; Métrich, 1985 : 238 ; Grieve, 1996 : 262 ; Nølke, 1991 : 218 ; Hansen, 2005a : 45-47), ainsi dans l'exemple suivant : Madame Lepic sert elle-même les enfants, d'abord grand frère Félix parce que son estomac crie la faim, puis sœur Ernestine pour sa qualité d'aînée, enfin Poil de Carotte qui se trouve au bout de la table (TLF). Ce sens est attesté depuis ca 1170 (RouH, livre II, vers 650$)^{5}$ :

Et li roiz et sa gent lez ont touz desconfiz, A plusors ont trenchiez et oreilles et piez ; Ne poent asseür reposer en lor liez, Destruit son tuit enfin, cescun n'en est respiez («Et le roi et ses troupes les [= les Anglais] ont tous mis en déroute, à plusieurs d'entre eux ils ont coupé les oreilles et les pieds; ils ne peuvent plus se reposer en sûreté dans leurs lits, et à la fin ils sont tous mis à mort, aucun d'eux n'est épargné »).

L'ensemble des corrélats romans de fr. enfin connaissent cette même valeur : sard. in finis (Poesie in dial. mer. [in finis, ti prego], DES s.v. fine ${ }^{1}$ ), it. infine (dp. 1294 [Guittone, Lettere in prosa : anima e Dio e amore e onore, e corpo infine], OVI ; DELI ${ }^{2}$; GDLI), frioul. infin (dp. 16 ${ }^{\mathrm{e}}$ s., Joppi, $1878: 241$ [in fine] ; Pirona, 1992 [infin c'o rîf uei fâ a miò mút]), occit. enfin (dp. 1460 [région d'Avignon], FEW 3, 561a; fin $15^{\mathrm{e}}$ s. [Mystère de Saint Pons] ; $15^{\mathrm{e}}$ s. [Istoria Petri \& Pauli : anfin]; COM), gasc. enfí (Palay), esp. enfin (1500/1520 [Castillejo, Reprensión contra los poetas españoles que escriben en italiano: Desprecian [...] usan ya de cierta prosa [...] tienen por simples [...] Dauan enfin aentender aquellos viejos autores no a ver sabido hazer buenos metros] - 1721 [Bluteau] ; CORDE ; NTLLE) et port. enfim «id. » (dp. $15^{\mathrm{e}}$ s. [Zurara, Crónica da Guiné : partindosse do seu começo e cótinuado prosseguimento atees que a elles se tornã em fy], Cunha, 2002 ; DLPC [ « por último, em último lugar », par exemple mas 
o que se conserva, a pouco e pouco estraga-se, envelhece, envilece, mingua e enfim se apaga] $)^{6}$. Il n'y a donc pas de doute que l'étymon protoroman présentait déjà ce sens.

On pourrait nous objecter que ce n'est pas cette valeur grammaticale que les données philologiques du domaine français attestent le plus anciennement, enfin4 récapitulatif (une des six valeurs pragmatiques de enfin) apparaissant un demi-siècle plus tôt dans les textes ( $c f$. ci-dessous 2.2.2). Néanmoins, cet état de fait ne constitue aucunement une contre-indication: les sens pragmatiques (procéduraux) sont par définition secondaires par rapport aux sens lexicaux et grammaticaux (référentiels) ( $c f$. Traugott \& Dasher, 2002 : 188-189), et une lacune documentaire, phénomène dont on n'est jamais à l'abri en linguistique historique, est à même d'expliquer le décalage chronologique constaté.

\subsubsection{Enfin2 aspectuel}

Un deuxième emploi de enfin peut être glosé par « finalement (indique qu'un procès se déroule après un long espace de temps) » (von Wartburg 1932 in FEW 3, 561a, FĪNIS I 1 ; TLF 1979 [I. B.] ${ }^{7}$; Cadiot et al., 1985 : 211-219 [2. B.] ; Métrich, $1985: 237$ et 239; Oguma, $1987: 73$; Franckel, 1989 : 124 ; Hansen, 2005a : 53-54 et 61). À la suite de M.-B. Hansen (2005a : 53), nous retenons, pour désigner ce enfin2, la dénomination de enfin aspectuel, celle de enfin de soulagement (Cadiot et al., $1985: 212$ ) nous paraissant moins pertinente: s'il est vrai que l'actualisation d'un procès après une longue attente provoque un sentiment de soulagement, cette implication psychologique nous semble secondaire ${ }^{8}$. Ce enfin 2 est actualisé par exemple dans Il regarda son fils pendant longtemps, et lui dit enfin d'une voix affaiblie: Ernest, mon enfant, tu es bien jeune; mais tu as bon cœur... (TLF). Ce marqueur aspectuel est attesté depuis ca 1175 (GautArrErR, vers 1843 = Hansen, 2005a : 46 [qui y voit enfin1, ce qui nous paraît représenter une lectio difficilior dans la mesure où l'on n'est pas en présence d'une série de procès] $)^{9}$ :

Preudom porte o lui son tesmoig; Nel puet empirier male bouce, Ne malvais hom a lui ne touce, Ne ja preudom n'ert por lui pire, Por canqu'il onques set mesdire, Car li mauvais, il s'en confont, Car li plons art et l'argent font, Et si s'afine par le plom, Qui s'art por lui; li mauvais hom Art enfin por le proude gent Si con li plons fait por l'argent («L'honnête homme garde toujours sa bonne réputation ; les propos malveillants n'y changent rien, l'homme méchant ne l'atteint même pas. L'honnête homme ne sera pas endommagé par lui, quoi qu'il dise. Car le mauvais se consomme lui-même : c'est comme le plomb qui brûle et l'argent qui fond et s'affine grâce au plomb qui brûle pour lui. Le mauvais homme finit par brûler pour les braves gens comme le plomb le fait pour l'argent »).

Cinq autres langues romanes présentent d'exacts correspondants de enfin2 aspectuel, dont trois attestés depuis le Moyen Âge : sard. in finis (Ulargiu [ma in finis narat], DES), it. infine (dp. $13^{\mathrm{e}} \mathrm{s}$. [Poi in fine un di loro andò con gli altri... ambasciatori a Carlo Magno imperatore per fare rifare la detta città di Firenze]; GDLI ; DELI²), frioul. infin (Pirona, 1992 [infin dai faz al à vût resòn lui]), esp. enfin (1344 [Crónica de 1344 : Et tornaron otra vez contra gulfayan \& lidiaron conel \& duroles la batalla quatro dias pero enfin vençio ahjran aquella vez \& traxo muy mal alos otros] - 1721 [Bluteau]; CORDE; NTLLE) et port. enfim (dp. $15^{\mathrm{e}} \mathrm{s}$. [Lopes, Crónica de D. Fernando : Sobr'esto ouve muitos conselhos, e enfim acordarom que a fosse cercar, pollas muitas viandas que n'aquella comarca avia], Cunha, 2002 ; DLPC [«em momento há muito esperado », par exemple vinha agarrar-me, apanhar-me como quem, enfim encontra alguém num deserto] $)^{10}$.

Ainsi, si nous suivons volontiers M.-B. Hansen (2005a : 54) pour considérer ce second sens comme issu de celui décrit sous 2.1.1, la comparaison avec les autres langues romanes nous incite néanmoins à supposer que ce sens est acquis dès le protoroman : il s'agit, en français, d'un sémantisme hérité et non pas d'une innovation. 


\title{
2.2 Pragmatème
}

\subsubsection{Enfin3 épistémique}

Parmi les six principales valeurs pragmatiques de enfin, la question d'un éventuel héritage du protoroman se pose pour une seule, à savoir pour la valeur épistémique (selon la terminologie de Hansen, 2005a : 50). Cet emploi de enfin pourrait être glosé par « en dernière analyse, tout compte fait, tout bien considéré (marque que le locuteur arrive à une conclusion sur la base de tous les éléments disponibles) » (TLF 1979 [II.3.] ; Luscher \& Moeschler, 1990 : 94-95 ; Barnes, 1995 : 820 ; Grieve, 1996 : 264 ; Hansen, 2005a : 50-51 [《the speaker is drawing a conclusion based on available evidence »]). Il est actualisé dans l'exemple suivant: Pourquoi n'est-ce pas à moi qu'on vous adresse? Mais je verrai bientôt si vous me convenez : car enfin, c'est à moi que vous appartenez, et vous êtes vraiment entrée à mon service (TLF). Ce enfin3 épistémique est attesté depuis ca 1185 (ProtH, vers 2604) ${ }^{11}$ :

\begin{abstract}
Dreit a l'issir d'une sapee [Melander] Ad choisi deus hommes erranz Qui portoent deus trussels granz. E cil reunt Melander veü, Esbaï sunt e esperdu... Ne ne surent quel part aller. Melander les en fait mener; Ço fu Jubar, od li Mathan [deux confidents de Protheselaus] ; Mult urent soffert peine e ahan, Dol e travail pur lor seinnur, Or sunt en brande e en poür. Melander les met a raison : Enfin, andui estes laron. La robe que avez ça porté Voil veer, kar ele est emblé (« Au sortir d'une forêt de pins, [Melander] voit deux hommes qui y portent deux grands coffres. Et ils voient Melander à leur tour, ils en sont effrayés et confus... et ne savaient pas où aller. Melander les a fait prendre ; c'était Jubar, et avec lui Mathan ; ils avaient beaucoup souffert pour leur seigneur, maintenant ils sont dans l'angoisse et ils ont peur. Melander leur demande des comptes : "Tout bien considéré, vous êtes des larrons. Je veux voir la robe que vous portez là, puisque vous l'avez volée"») $)^{12}$.
\end{abstract}

Le français partage la valeur épistémique avec l'italien, où elle est attestée de même depuis le Moyen Âge, et de façon ponctuelle avec l'espagnol du $16^{\mathrm{e}}$ siècle: it. infine (dp. 1268 [Trattati morali di Albertano da Brescia volgarizzati: schifare de' la guerra quantunque tu pòi [...], per molte ragione. La prima è [...]. La seconda ragione [...]. La sesta ragione [...]. Et all'ultimo ti dico; che infine tre ragion sono per le quale battaglie non si potrebbero agevolmente pensare], OVI ; DELI $^{2}$ [dp. 1543]; GDLI [dp. 1543]), esp. enfin (ca 1539 [Villalón, El Scholástico: Todas quieren mandar, y que las obedezcamos : quieren ser libres, y nos otros sus captiuos: quieren hablar, y que todos callemos: enfin quieren ser señoras absolutas y por tales ser estimadas], CORDE). Étant donné le caractère ténu de ces parallèles d'une part, les ouvrages de référence datent l'apparition du pragmatème italien du $16^{\mathrm{e}}$ siècle seulement, d'autre part, l'existence du pragmatème espagnol est déduite d'un hapax -, la prudence exige toutefois de considérer, en l'état actuel de nos connaissances, que enfin et ses congénères ont basculé de façon indépendante du domaine de la grammaire à celui de l'énonciation. On rejoint donc là le cas d'une pragmaticalisation parallèle dans différentes langues romanes tel qu'il a été mis en évidence par R. Waltereit (2006a) pour it. guarda, fr. regarde, esp. mira et port. olha.

Pour ce qui est de l'origine de enfin3 épistémique, les résultats de notre étude tendent à départager les deux hypothèses envisagées par M.-B. Hansen (2005a : 63), qui hésite entre un rattachement à enfin 1 temporel et enfin4 récapitulatif, en faveur de enfin 1 temporel, et cela en dépit de la chronologie (enfin3 est attesté plus d'un demi-siècle après enfin4, contingence que nous mettons sur le compte d'une documentation insuffisante), car l'italien, qui partage clairement enfin3 avec le français, ne possède pas l'équivalent de enfin4.

\subsubsection{Enfin4 récapitulatif}

La deuxième valeur pragmatique de enfin peut être rendue par «bref, en un mot (marque que le locuteur conclut, résume à la fin d'un développement) »(Hosch, 1896: 10; TLF 1979 [II.A.]; Cadiot et al., 1985 : 205-207 [2.A.1.b.] et 210-211 [2.A.2.b.] ; Métrich, $1985: 238$ et 240 ; Franckel, $1989: 125 ; 129$ [« reprise synthétique »] ; Luscher \& Moeschler, 1990 : 95-96 ; Grieve, 1996 : 264 ; Hansen, 2005a : 47- 
$\left.50 ; 52-53^{13}\right)$. On la trouve par exemple dans l'énoncé suivant: Ne m'a-t-il pas appris la musique, le dessin, la grammaire, enfin tout ce que je sais? (TLF). Ce enfin4 récapitulatif est attesté depuis 1119 $(\text { PhThCompS, vers } 2701=\mathrm{TLF})^{14}$ :

\begin{abstract}
Et ore vëez raisun Que eclipsium apellum. Ceo est 'devisement', Sulum romaine gent, De lumere en vertéd, Si cum est espruvéd. Ceo dit Plinïus, Ki ert bons philosophus. Iceo sacez en fin : Iceo est eclipsin Del solail, quant la lune Nus fait si grant ra[n]cune Que ele est tant alee Qu'ele ad acuveitee Tute terre üelement ; Eissifaiterement Que li solails li vent Dreit desus, dunc retent Sa clartéd, sa lüur, Fait nus grant tenebrur («Et maintenant vous allez voir un phénomène que nous appelons éclipse. Cela veut dire, en réalité, la division, selon les gens qui parlent en langue romane, de la lumière, comme c'est prouvé. C'est Pline qui l'a dit, et il était un grand érudit. Bref, sachez ceci : l'éclipse de soleil, c'est quant la lune nous fait un grand désagrément par le fait qu'elle a tant avancé qu'elle a couvert toute la terre d'une manière égale ; de la sorte que le soleil vient directement derrière elle et retient donc sa clarté et sa lumière, et nous fait une grande obscurité »).
\end{abstract}

On relève des parallèles de enfin4 récapitulatif en italien, en espagnol et en portugais : it. in fino (1455 [Cronaca di Pietro Angelo di Gioavanni : le rede comenzaro a trare li sassi a li detti birri e li birri a loro, e così se comenzzò a gridare - A li sassi a li birri ! - di modo che in poco de ora se smantellaro più de 300 persone per trare e rencastelarli dentro, in fino el romore era grande], Folena \& Migliorini, 1953 : 74), esp. enfin (ca 1539 [Villalón, El Scholástico: Son todas pesadas, moledoras, pedigueñas, auarientas, luxuriosas, parleras, astutas, sagaçes, arteras, embaidoras, encantadoras, hechizaras : enfin inuentoras de toda maldad engaño y traiçion], CORDE ; 1706 [Stevens : " in short »], NTLLE), port. enfim (DLPC [ " (usa-se para introduzir uma conclusão final ou uma síntese) », par exemple gritou, espernenou, chorou, enfim, fez uma cena et « (usa-se para acrescentar um último elemento, por vezes globalizante, a uma enumeração)», par exemple na pobreza, no contraste social, no desemprego, na delinquência, no crime, enfim em tudo]). Le caractère tardif de ces parallèles incite toutefois à les mettre sur le compte de développements indépendants.

Comme le montre M.-B. Hansen (2005a : 48-50), enfin4 récapitulatif se rattache à enfin 1 temporel.

\title{
2.2.3 Enfin5 énumératif
}

Tandis que les deux premières valeurs pragmatiques de enfin sont attestées dès l'ancien français, la troisième ${ }^{15}$ n'apparaît qu'en moyen français, de sorte qu'il s'agit sans conteste d'une innovation française. On rendra la charge énonciative de enfin5 par «citons pour terminer (marque le dernier élément d'une énumération) » (von Wartburg 1932 in FEW 3, 561a, FİNIS I 1 ; TLF 1979 [I.C.] ${ }^{16}$; Métrich, 1985 : 238 ; Franckel, 1989 : 125 ; 129 ; Nølke, 1991 : 218-219; Rossari, 1997 : 28 ; Hansen, 2005a : 47), ainsi dans l'exemple suivant: Il y a à Paris trois polices : primo : la police du royaume [...] secondo : celle du régent [...] enfin celle de Dubois (TLF). Cette valeur est attestée depuis ca 1400 (EvrartContyEchG, page $245=\mathrm{DMF} 2$ s.v. barater; autre occurrence s.v. rapairier $)^{17}$ :

[...] ce sont troiz vices et troiz maulx qui voulentiers repairent en amours et ly font compaignie, c'est assavoir luxure et avarice et trayson ou infidelité ; luxure donc y est pour deliter, et avarice y est pour profiter, et trayson enfin pour l'amant bareter. («[...] il existe trois vices ou maux qui cohabitent volontiers avec l'amour et lui tiennent compagnie, à savoir la luxure, l'avarice et la trahison ou infidélité ; la luxure sert à charmer, l'avarice à profiter, enfin la trahison, à tromper son amant »).

Ce enfin5 énumératif se rattache à enfin 1 temporel (Hansen, 2005a : 48-49) : le sémantisme passe de la sphère mondaine («dernier élément d'une série de procès ») à la sphère discursive («dernier élément d'une énumération »). 


\subsubsection{Enfin6 rectificatif}

La valeur de enfin la plus fréquemment réalisée en français oral spontané contemporain (Beeching, 2002 : 127) peut être glosée par «du moins (indique une rectification, un amendement) » (TLF 1979 [II.2.] ; Cadiot et al., 1985 : 230-235 [3.B.2.a. et 3.B.2.b.] ; Métrich, $1985: 238$ et 240 ; Franckel, 1989 : 124125 ; 129-130 ; Nølke, 1991 : 219 ; Hwang, 1993 : 47-48 [qui distingue auto-rectificatif et hétérorectificatif] ; Barnes, 1995 : 820 ; Grieve, 1996 : 264 ; Rossari, 1997 : 28-33 ; 2000 : 105-109 ; Beeching, 2001 : 27-38 ; 2002 : 130-144 ; Hansen, 2005a : 58-59 et $62^{18}$ ). Actualisé dans l'exemple suivant : Autour de vous, vos camarades sont tous pareils? - Tous... enfin, tous ceux qui existent, qui réfléchisssent (TLF), enfin6 rectificatif est attesté depuis 1696 (Sévigné, Correspondance, Frantext = Hansen, 2005a : $58)^{19}$ :

Je crois être dans un autre climat, un pays bas et couvert comme la Bretagne, enfin sombre forêt où le soleil ne luit que rarement.

À en croire le témoignage muet des manuels de conversation du $17^{\mathrm{e}}$ siècle, dont les dialogues ne contiennent aucune trace de ce enfin6 rectificatif (Radtke, 1994 : 281), la datation proposée par M.-B. Hansen sera probablement difficile à repousser. En tout état de cause, le parallèle fonctionnel que constitue port. enfim (DLPC ["(usa-se para arescentar algo que expressa com maior rigor o que se pretende indicar) », par exemple se me formei um pouco, enfim se estudei, foi à custa do meu pai]) doit clairement être considéré comme génétiquement indépendant.

L'apparition de enfin6 peut s'expliquer par un glissement de sens soit à partir de enfin 1 temporel, soit à partir de enfin4 récapitulatif (Hansen, 2005a : 58 ; 63). Après avoir passé en revue un grand nombre d'occurrences dans le but de dater enfin6, nous opterions plus volontiers pour enfin4 récapitulatif, car dans un nombre non négligeable d'attestations, nous hésitions entre une interprétation en enfin4 et enfin6 avant de pouvoir trancher.

\subsubsection{Enfin7 performatif}

Une glose possible de enfin 7 performatif, appellation que nous empruntons à F. Nemo (2000:501), serait « oublions cela !, tant pis ! (indique que le locuteur renonce à insister) » (Hosch, 1896: 10; TLF 1979 [II.B.2.] ; Cadiot et al., 1985 : 208-210 [2.A.2.a.] et 228-229 [3.B.1.]; Métrich, 1985 : 240 ; Franckel, 1989 : 125 ["clôture d'une conversation en suspens »] ; 130 ; Nemo, 2000 : 500-501; Hansen, 2005a : 59-60; Paillard, $2003: 400)^{20}$. Cette valeur de enfin, qui s'apparente à la prétérition (Cadiot et al., 1985 : 209), est actualisée dans l'exemple suivant : ... et quant à ma pauvre Virginie, elle veut aller vivre dans notre château de Lorraine, chez mon père, avec son tourment. Enfin! ... Elle a son petit pour la consoler! (TLF). Elle est attestée depuis 1703 (Saint-Évremond, Les Académiciens, Frantext = Hansen, 2005a : 60$)^{21}:$

\footnotetext{
Je voudrois que Gombauld, L'Estoille et Colletet, En prose comme en vers eussent un peu mieux fait, Que des Amis rivaux Boisrobert ayant honte, Revînt à son talent de faire bien un conte. Enfin... [fin du tour de parole].
}

Parmi les langues sœurs du français, seul le portugais présente l'équivalent de cette valeur (DLPC s.v. enfim [ " (expressão usada para indicar tolerância ou aceitação com o que foi dito ou com a situação, apesar de não se concordar completamente) », par exemple arranje-me dois gatos - um preto e outro branco - porque também dâ. - $\hat{E}$ uma altenativa sobremaneira insólita, mas, enfim]).

Ce enfin 7 performatif pourrait remonter soit au enfin 6 rectificatif, soit au enfin 8 réprobateur (Hansen, 2005a : 59-60). Si notre préférence va vers enfin6 rectificatif, c'est que enfin7 performatif nous semble avoir gardé une charge sémantique « annulation de ce qui vient d'être dit».

\subsubsection{Enfin8 réprobateur}

Un dernier emploi énonciatif de enfin peut être rendu par « voyons ! (marque une impatience réprobatrice, un rappel à l'ordre) » (TLF 1979 [II.B.3. : 'fam.' et II.B.4.] ; Cadiot et al., 1985 : 220-227 [3.A.1.] ; 
Métrich, 1985 : 238 et 240; Franckel, 1989: 124; 130-131; Grieve, 1996: 263 ; $c f$. Luscher \& Moeschler, 1990 : 93-94; Hansen, 2005a : 59 [impatient dismissal] et 61). On appellera cet emploi enfin8 réprobateur; il apparaît par exemple dans l'énoncé suivant: Enfin, c'est inadmissible : qu'est-ce que vous fabriquez ici à cette heure? (TLF). La première attestation présentant cette valeur remonte à 1756 (Gueullette, Léandre fiacre, Frantext = Hansen, 2005a : 59) ${ }^{22}$ :

Gilles : Oui, elle est belle ; mais un homme qui a une belle femme, tout le monde est son cousin.

Cassandre : Hé bien tant mieux, on me fera plus d'honneur.

Gilles: Mais pardienne, ne savez-vous donc pas qu'une bonne chèvre, une bonne mule, et une bonne femme sont trois mauvaises bêtes?

Cassandre : Enfin, je te demande conseil, mais ce n'est pas pour me contredire.

Ce enfin8 réprobateur se rattache à enfin4 récapitulatif (Hansen, 2005a : 59).

\section{Résultats}

Le graphique ci-après récapitule l'échelonnement diachronique des différentes valeurs, grammaticales et pragmatiques, du marqueur enfin du français contemporain.

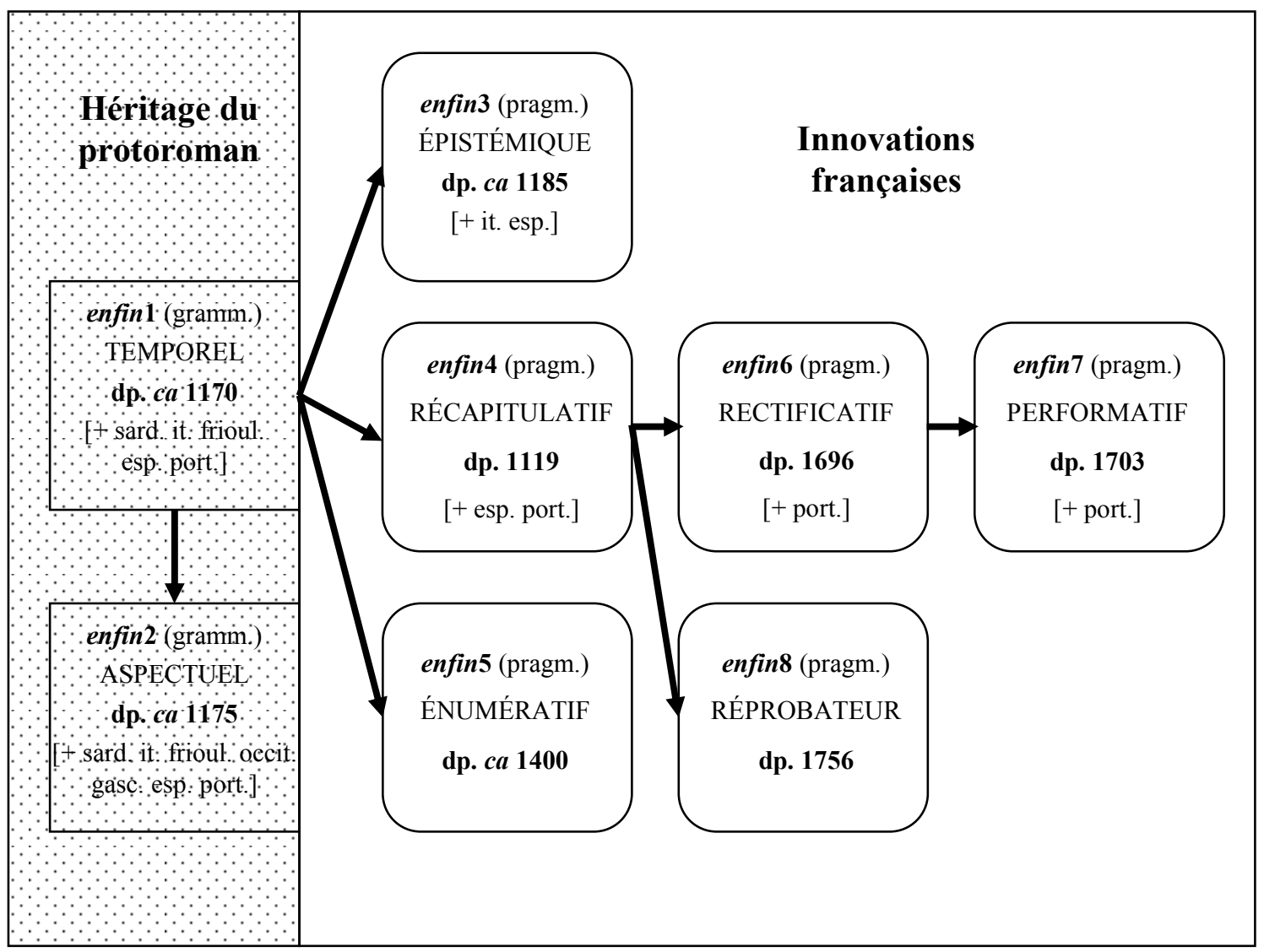

Graphique : reconstruction de l'agencement diachronique de la polysémie du marqueur enfin du français contemporain 


\section{Conclusion}

$\mathrm{Au}$ terme de cette étude, il convient de s'interroger sur le gain en connaissances nouvelles qui s'en dégage. On citera en premier lieu les apports concrets à la description historique du marqueur grammatical et pragmatique enfin, à commencer par quatre antédatations : de cinq ans pour enfin 1 , de près de 190 ans pour enfin 5 , de plus de 350 ans pour enfin 3 , voire de plus de 450 ans pour enfin 2 (à noter que par rapport au FEW, ce sont des rétrodatations que nous proposons, de 25 ans pour enfin 2 et de 250 ans pour enfin5). Faisant levier sur ces datations renouvelées, mais aussi et surtout sur la comparaison romane, nous en sommes venus à proposer un scénario passablement transformé de la pragmaticalisation de enfin. De façon quelque peu inattendue, ce travail nous a incités en outre à proposer une étymologie inédite pour le grammème enfin: il ne s'agirait pas d'un composé formé en français, mais d'un continuateur du protoroman.

À un niveau plus général, étant donné que les travaux consacrés aux marqueurs pragmatiques qui adoptent un point de vue diachronique sont encore relativement rares $(c f \text {. Hansen \& Rossari, } 2005: 181)^{23}$ et que l'approche génético-comparative adoptée ici est inédite en pragmatique, nous espérons avoir apporté aussi une contribution d'ordre méthodologique au débat sur la genèse des marqueurs du discours.

Mais on ne saurait s'arrêter là : il serait intéressant à présent de mettre en rapport nos résultats avec la genèse des pragmatèmes complexes sur enfin ( $c f$. Métrich, 1985: 241), dont notamment enfin bon (Bertrand \& Chanet $2005: 51 ; 58-59)$, enfin bref (Grieve, $1996: 264$; Waltereit, $2007: 106-108$ ) ainsi que mais enfin (Hosch, $1896: 10$; Grieve, $1996: 263$; Razgouliaeva, 2002 ; Hansen, 2005a : 55-56) et sa variante m'enfin (Barnes, $1995: 819-820$ ). La question de cette polypragmaticalisation (Dostie 2004) mériterait d'être abordée dans une prochaine publication.

\section{Références bibliographiques}

Bambeck, M. (1958). Miszellen. 1. ALTERUM TALEM ; 2. IN FINE. Romanische Forschungen, 70, 126-128.

Barnes, B. K. (1995). Discourse Particles in French Conversation : (eh) ben, bon, and enfin. The French Review, 68, 813-821.

Beeching, K. (2001). Repair strategies and social interaction in spontaneous spoken French : the pragmatic particle enfin. Journal of French Language Studies, 11, 23-40.

Beeching, K. (2002). Gender, Politeness and Pragmatic Particles in French. Amsterdam/Philadelphia : Benjamins.

Beeching, K. (2007). La co-variation des marqueurs discursifs bon, c'est-à-dire, enfin, hein, quand même, quoi et si vous voulez : une question d'identité ? Langue française, 154, 78-93.

Bertrand, R. \& Chanet, C. (2005). Fonctions pragmatiques et prosodie de enfin en français spontané. Revue de Sémantique et Pragmatique, 17, 41-68.

Buchi, É. (2007a). Sur la trace de la pragmaticalisation de l'adverbe toujours (« Voyons toujours l'apport de la linguistique historique »). Langue française, 154, 110-125.

Buchi, É. (2007b). Approche diachronique de la (poly)pragmaticalisation de français déjà («Quand le grammème est-il devenu pragmatème, déjà ? »). In : Trotter, D. (éd.) : Actes du XXIV Congrès international de linguistique et de philologie romanes (Aberystwyth 2004). Tübingen : Niemeyer : $3: 251-264$.

Buchi, É. (à paraître). Le passage de la sphère grammaticale à la sphère énonciative de l'adverbe français encore du point de vue de la linguistique historique («C'est encore dans ce domaine-là qu'il y a la plus grande marge de progression »). In : [Volume de mélanges en l'honneur d'un collègue].

Cadiot, A. \& Ducrot, O. \& Fradin, B. \& Nguyen, Th. B. (1985). Enfin, marqueur métalinguistique. Journal of Pragmatics, 9, 199-239.

Cioranescu = Cioranescu, A. (1966). Diccionario etimológico rumano. Tenerife : Biblioteca Filológica.

COM = Ricketts, P. T. (2005). Concordance de l'occitan médiéval. Turnhout : Brepols. 
CORDE = Real Academia Española (2002-). Corpus Diacrónico Español (CORDE). Madrid : Real Academia Española : site Internet (http://corpus.rae.es/cordenet.html).

Cunha, A. G. da (dir.) (2002). Vocabulário Histórico-Cronológico do Português Medieval (DVD). Rio de Janeiro : Fundação Casa de Rui Barbosa.

DEAF = Baldinger, K. et al. (1971-). Dictionnaire étymologique de l'ancien français. Québec, Tübingen \& Paris : Université de Laval, Niemeyer \& Klincksieck.

$\mathrm{DELI}^{1 / 2}=$ Cortelazzo, M. \& Zolli, P. (1979-1988/1999²). Dizionario Etimologico della Lingua Italiana. Bologne : Zanichelli.

DELP $^{3}=$ Machado, J. P. (1977 $\left.\left[1952^{1}\right]\right)$. Dicionario etimológico da lingua portuguesa com a mais antiga documentação escrita e conhecida de muitos dos vocábulos estudados (5 vol.). Lisbonne : Horizonte.

$\mathrm{DENF}^{2}=$ Cunha, A. G. da $\left(1986^{2}\left[1982^{1}\right]\right)$. Dicionário etimológico Nova Fronteira da língua portuguesa. Rio de Janeiro : Nova Fronteira.

DES = Wagner, M. L. (1960-1964). Dizionario etimologico sardo (3 vol.). Heidelberg : Winter.

DEX $^{2}=$ Academia Română $\left(1996^{2}\left[1975^{1}\right]\right)$. DEX. Dicționarul explicativ al limbii române. Bucarest: Univers Enciclopedic.

DLPC = Academia das Ciências de Lisboa (2001). Dicionário da língua portuguesa contemporânea (2 vol.). Lisbonne: Verbo.

DMF2 = ATILF (2007-). Dictionnaire $d u$ Moyen Français (DMF2). Nancy: ATILF : site Internet (http://stella.atilf.fr/gsouvay/dmf2 ; $c f$. http://www.atilf.fr/dmf pour DMF1).

Dostie, G. (2004). Pragmaticalisation et marqueurs discursifs. Analyse sémantique et traitement lexicographique. Bruxelles : De Boeck \& Duculot.

Espinas, G. \& Pirenne, H. (1920/1924). Recueil de documents relatifs à l'histoire de l'industrie drapière en Flandre : $l^{\text {ère }}$ partie : Des origines à l'époque bourguignonne (2 vol.). Bruxelles : Kiessling.

FEW = Wartburg, W. von et al. (1922-2002). Französisches Etymologisches Wörterbuch. Eine darstellung des galloromanischen sprachschatzes (25 vol.). Bonn/Berlin/Bâle : Klopp/Teubner/Zbinden.

Folena, G. \& Migliorini, B. (éd.) (1953). Testi non toscani del Quattrocento. Modène : Società tipografica Modenese.

Franckel, J.-J. (1989). Étude de quelques marqueurs aspectuels du français. Genève : Droz.

Frantext $=$ ATILF (2002-). Frantext. Outil de consultation de ressources informatisées sur la langue française. Nancy : ATILF : site Internet (http://www.frantext.fr).

GDIU = De Mauro, T. (dir.) (1999/2000). Grande dizionario italiano dell'uso (6 vol.). Turin : UTET.

GDLI = Battaglia, S. (1961-2004). Grande dizionario della lingua italiana (22 vol.). Turin : UTET.

Grieve, J. (1996). Dictionary of Contemporary French Connectors. Londres/New York : Routledge.

Hansen, M.-B. Mosegaard (2005a). From prepositional phrase to hesitation marker. The semantic and pragmatic evolution of French enfin. Journal of Historical Pragmatics, 6, 37-68.

Hansen, M.-B. Mosegaard (2005b). A comparative study of the semantics and pragmatics of enfin and finalement, in synchrony and diachrony. Journal of French Language Studies, 15, 153-171.

Hansen, M.-B. Mosegaard \& Rossari, C. (2005). The evolution of pragmatic markers. Introduction. Journal of Historical Pragmatics, 6, 177-187.

Hosch, S. (1895-1897). Französische Flickwörter. Ein Beitrag zur französischen Lexikographie (3 vol.). Berlin : Gaertner.

Houaiss = Houaiss, A. et al. (2001). Dicionário Houaiss da língua portuguesa . Rio de Janeiro : Objetiva.

Hwang, Y. (1993). Eh bien, alors, enfin et disons en français parlé contemporain. L'information grammaticale, 57, $46-48$.

Joppi, V. (1878). Testi inediti friulani dei secoli XIV a XIX. Archivio Glottologico Italiano, 4, 185-342. 
Latham = Latham, R. E. (1975-). Dictionary of Medieval Latin from British Sources. Londres : Oxford University Press.

Luscher, J.-M. \& Moeschler, J. (1990). Approches dérivationnelles et procédurales des opérateurs et connecteurs temporels : les exemples de et et de enfin. Cahiers de linguistique française, 11, 77-104.

Métrich, R. (1985). Propositions pour le traitement des «mots du discours » en lexicographie bilingue françaisallemand : l'exemple de d'accord, donc et enfin (Troisième volet : enfin). Nouveaux Cahiers d'Allemand, 3, 237247.

Nemo, F. (2000). Enfin, encore, toujours entre indexicalité et emplois. In : Englebert, A. et al. (éd.) : Actes du XXII Congrès International de Linguistique et de Philologie Romanes (Bruxelles, 23-29 juillet 1998). Tübingen : Niemeyer : vol. $7: 499-511$.

Nølke, H. (1991). Contrastive Pragmatic Linguistics. In : Lauridsen, K. M. \& Lauridsen, O. (éd.) : Contrastive Linguistics. Papers from the CL-symposion, 28-30 August 1989 The Aarhus School of Business. Aarhus: Udgiverne : 199-231.

Nølke, H. (2006). Petite étude diachronique de or. De la deixis temporelle à la deixis textuelle. In : Nølke, H. et al. (éd.) : Grammatica. Festschrift in honour of Michael Herslund. Berne : Lang : 393-404.

NTLLE = Real Academia Española (2001). Nuevo tesoro lexicográfico de la lengua española (2 DVD). Madrid: Espasa Calpe.

Oguma, K. (1987). Finalement/enfin/après tout [article en japonais]. Bulletin d'Études de Linguistique Française [Tokyo], 21, 72-74.

OLD = Glare, P. G. W. et al. (1968-1982). Oxford Latin Dictionary. Oxford : Clarendon.

OVI = Istituto Opera del Vocabolario Italiano (2001-). OVI (Opera del Vocabolario Italiano). Florence : CNR \& Accademia della Crusca : site Internet (http://www.ovi.cnr.it).

Paillard, D. (1998). Les mots du discours comme mots de la langue. Le gré des langues, 14, 10-41.

Paillard, D. (2003). À propos de enfin. In : Combettes, B., Schnedecker, C. \& Theissen, A. (éd.) : Ordre et distinction dans la langue et le discours. Actes du Colloque international de Metz (18, 19, 20 mars 1999). Paris : Champion : 387-408.

Palay = Palay, S. $\left(1980^{3}\left[1932^{1}\right]\right)$. Dictionnaire du béarnais et du gascon modernes (bassin aquitain) embrassant les dialectes du Béarn, de la Bigorre, du Gers, des Landes, et de la Gascogne maritime et garonnaise. Paris: Éditions du CNRS.

Pirona, G. A. et al. (1992 $\left.{ }^{2}\left[1967^{1}\right]\right)$. Il nuovo Pirona. Vocabolario friulano. Udine : Società Filologica Friulana.

Radtke, E. (1994). Gesprochenes Französisch und Sprachgeschichte. Zur Rekonstruktion der Gesprächskonstitution in Dialogen französischer Sprachlehrbücher des 17. Jahrhunderts unter besonderer Berücksichtigung der italienischen Adaptionen. Tübingen : Niemeyer.

Razgouliaeva, A. (2002). Combinaison des connecteurs mais enfin. Cahiers de linguistique française, 24, 143-168.

Reichenberger, K. (1956). Lat. ALTERUM TANTUM. Romanische Forschungen, 68, 147-148.

Rossari, C. $\left(1997^{2}\left[1994^{1}\right]\right)$. Les Opérations de reformulation. Analyse du processus et des marques dans une perspective contrastive français-italien. Berne et al. : Lang.

Rossari, C. (2000). Connecteurs et relations de discours : des liens entre cognition et signification. Nancy : Presses Universitaires de Nancy.

Smith, J. S. (2006). From adverb to discourse marker and beyond. The status of là in franco-american French. In : Gess, R. S. \& Arteaga, D. (éd.) : Historical Romance Linguistics. Retrospective and perspectives. Amsterdam/Philadelphie : Benjamins : 375-387.

TLF = Imbs, P. \& Quemada, B. (dir.) (1971-1994). Trésor de la Langue Française. Dictionnaire de la langue du $X I X^{e}$ et du XX siècle (1789-1960) (16 vol.). Paris : Éditions du CNRS/Gallimard.

Traugott, E. Closs \& Dasher, R. B. (2002). Regularity in semantic change. Cambridge : Cambridge University Press. 
Waltereit, R. (2006a). Comparer la polysémie des marqueurs du discours. In : Drescher, M. \& Frank-Job, B. (éd.) : Les Marqueurs discursifs dans les langues romanes. Approches théoriques et méthodologiques. Francfort : Lang : 141-151.

Waltereit, R. (2006b). The rise of discourse markers in Italian : a specific type of language change. In : Fischer, K. (éd.) : Approaches to Discourse Particles. Amsterdam et al. : Elsevier : 61-76.

Waltereit, R. (2007). À propos de la genèse diachronique des combinaisons de marqueurs. L'exemple de bon ben et enfin bref. Langue française, 154, 94-109.

1 Nos remerciements les plus chaleureux s'adressent à Jean-Pierre Chambon (Université de Paris-Sorbonne), à JeanPaul Chauveau (ATILF) et à Frankwalt Möhren (DEAF), qui ont enrichi nos vues par leurs critiques perspicaces ; à l'équipe du Lessico etimologico italiano (Université de la Sarre), qui a, avec une grande amabilité, mis à notre disposition son fichier de matériaux du domaine italoroman ; à Takeshi Matsumura (Université de Tokyo), qui a bien voulu traduire l'article de Oguma (1987); à Johannes Staub (Académie des Sciences de Munich), qui a mis à notre disposition des matériaux inédits du Mittellateinisches Wörterbuch.

2 Les sigles bibliographiques sont ceux du DEAF : AmYdR = Reinhard, J. R. (éd.) (1926). Amadas et Ydoine. Paris : Champion ; ChrPisFaisS = Solente, S. (éd.) (1936/1940). Le Livre des faits et bonnes meurs du sage roy Charles $V$ par Christine de Pisan (2 vol.). Paris : Société de l'histoire de France ; DeschQ = Queux de Saint-Hilaire, P. A. \& Raynaud, G. (éd.) (1878-1903). Euvres complètes de Eustache Deschamps (10 vol.). Paris: Firmin Didot; EvrartContyEchG = Guichard-Tesson, F. \& Roy, B. (éd.), 1993. Le Livre des eschez amoureux moralisés, Evrart de Conty. Montréal : CERES ; GautArrErR = Raynaud de Lage, G. (éd.) (1976). Gautier d'Arras, Éracle. Paris : Champion; GuillPalMa = Micha, A. (1990). Guillaume de Palerne. Genève : Droz; NoomenFabl = Noomen, W. \& Boogaard, N. H. J. van den et al. (éd.) (1983-1998). Nouveau recueil complet des fabliaux (10 vol.). Assen : Van Gorcum; PhThCompS = Short, I. (1984). Philippe de Thaon. Comput. Londres : Anglo-Norman Text Society; RouH = Holden, A. J. (1970-1973). Le Roman de Rou de Wace (3 vol.). Paris : Picard ; TristBérG = Gregory, S. (éd.) (1992). The romance of Tristran by Beroul. Amsterdam/Atlanta : Rodopi. En outre, le signe Ø signifie que l'ouvrage en question n'apporte rien au sujet, et le signe * marque les formes reconstruites.

3 Le roumain connaît aussi în fine loc. adv. " enfin », mais il s'agit là (comme dans le cas du substantif fine) d'un gallicisme ou d'un italianisme récent et livresque (DEX ${ }^{2}$; Cioranescu).

4 Cela vaut pour l'italien («giustapposizione di in e fine », DELI ${ }^{1}$; «comp. per giustapposizione, da in e fine », GDLI ; «dalla loc. in fine », GDIU), le français, l'occitan et le gascon («Zuss. » [“composés"], von Wartburg in FEW 3, 560b, FINIS I 1 ; « dér. de fin*; préf. $e n^{*}$ », TLF) et le portugais («prep. em + fim », Houaiss ; « de em + fim », DLPC). Les correspondants sarde, frioulan et espagnol ne bénéficient pas d'un traitement étymologique.

5 Ce sens étymologique n'est daté ni par le FEW ni par le TLF ; Hansen, 2005a : 46 : ca 1175 ( $c f$. ci-dessous 2.1.2).

$6 C f$. en outre lat. in fine loc. adv. "à la fin (marque le dernier procès d'une série) » ( $2^{\mathrm{e}} \mathrm{s}$. [Pasteur d'Hermas : hilaris autem in fine erat et super subsellium sedens], Reichenberger, $1956: 148, \mathrm{n} .2 ; 4^{\mathrm{e}} / 5^{\mathrm{e}} \mathrm{s}$. [saint Augustin : Quare post finitam orationem non aliquid nobis inde tractavit, vel quod a capite posuit, vel quod in fine conclusid, vel quod in medio collocavit? et quod habet sententia in capite, hoc habet in fine], Bambeck, $1958: 128)$ et lat. méd. in fine loc. adv. «id. » (av. 1234 [Roger of Wendover, Flores Historiarum], Latham 1, 948a s.v. finis $5^{\circ}$ : per hoc... consilium ... amisit... rex Johannes primo corda gentis ssue, ... et post alias terras, et in fine totum thesaurum exhausit).

7 Nous considérons comme équivalent ce que le TLF met à part comme une valeur affective sous un paragraphe II.B.1. : " pour marquer la fin d'une longue attente ou recherche » (exemple : Vous voilà tous enfin !) ; cf. Luscher \& Moeschler, $1990: 90-91$.

8 On pourrait éventuellement envisager de subdiviser cette valeur en deux sous-valeurs, selon que l'aspect temporel ou l'aspect émotif est plus ou moins proéminent. Si nous y avons renoncé, c'est que dans les occurrences qu'il nous a été possible d'analyser, les deux aspects sont toujours présents simultanément.

9 FEW : dp. ca 1150 (datation non utilisable dans notre contexte, car von Wartburg mêle deux valeurs qui nous semblent à distinguer : « [adverbe qui sert à marquer que l'on conclut après une énumération ou qu'une chose arrive après s'être fait attendre] »); TLF : dp. 1636; Hansen, 2005a: 53: dp. 1627. - On observe une tradition ininterrompue de l'emploi aspectuel à partir du $12^{\mathrm{e}}$ siècle $: 4^{\mathrm{e}}$ quart $12^{\mathrm{e}}$ siècle (TristBérG, vers 298 : [Le roi, qui a observé la rencontre entre Tristran et Iseut, descend de l'arbre où il s'était caché et se dit à lui-même :] Or puis je bien enfin savoir [« À présent je peux finalement être certain »]; $4^{\mathrm{e}}$ quart $13^{\mathrm{e}}$ siècle (NoomenFabl, vol. $2, \mathrm{n}^{\mathrm{o}} 10, \mathrm{~ms}$. A, 
vers 260 : [Robert s'est déchargé l'intestin; sa femme lui demande :] Or estes vous garis enfin? [" êtes-vous finalement guéri ?»]).

$10 C f$. en outre lat. in fine $\left(1^{\mathrm{er}}\right.$ s. av. J.-Chr. [Velleius Paterculus : cum esset in fine bellum piraticum]; $1^{\mathrm{er}}$ s. [Silius Italicus : nunc animas tibi... paucas in fine enumerasse paro]; OLD s.v. finis $\mathrm{n}^{\mathrm{o}} 12 \mathrm{~b} ; 4^{\mathrm{e}} / 5^{\mathrm{e}} \mathrm{s}$. [saint Augustin : Intelligamus ergo vocem huius hominis, in cuius corpore unus sumus homo; et ibi videbimus ista vera bona Jerusalem. Sic enim dixit in fine: "Et videas bona quae sunt Jerusalem” et exposuit in fine dicens : (...) ad extremum («finalement ») ne quis dicat], Bambeck, $1958: 128$ ).

11 Hansen, 2005a : 50-51 : dp. 1544 ; TLF : dp. 1792. - Il ne s'agit pas là d'un témoignage isolé ; $c f$. aussi les attestations suivantes, du $13^{\mathrm{e}} \mathrm{et} \mathrm{du} 14^{\mathrm{e}}$ siècle : [Un loup-garou entreprend de se procurer à boire :] .I. Clerc encontre a .i. prouvoire Qui li portoit a sa maison.I. barisel de vin moult bon; Mais, je quit bien, n'en goustera Li provoires qui le manda. Quant li clers voit le leu venir, Ne set en fin que devenir, Tout rue jus quanqu'il portoit, En fuies torne a tel esploit Comme cil qui ne cuide mie Que il em puist porter la vie ( Un clerc rencontre un prêtre qui lui apporte un petit baril de très bon vin à la maison. Mais je crois bien que le prêtre, qui a commandé le vin, ne le goûtera pas. Quant le clerc voit le loup s'approcher, il ne sait pas, tout bien considéré, ce qu'il adviendra de lui ; il jette tout ce qu'il avait sur lui et il prend la fuite comme quelqu'un qui ne pense pas survivre », début $13^{\mathrm{e}} \mathrm{s}$., GuillPalMa, vers 3340); Les mariez pour leurs enfans se font Las et chetis; li marchant ensement Et laboureur ont moult de froissement, Et sont enfin pour leur avoir maudiz ( Les parents se donnent toute la peine du monde pour leurs enfants ; les marchands et les laboureurs ont beaucoup d'ennuis et sont, en fin de compte, maudits à cause de leurs biens ", $3^{\mathrm{e}}$ tiers $14^{\mathrm{e}}$ s., DeschQ, vol. 1, p. 176)

12 Une attestation postérieure d'un siècle nous donnera l'occasion d'insister sur l'importance de la position syntaxique de enfin. On relève en effet dans les manuscrits T et C (datés de ca 1300) du Bouchier d'Abeville (NoomenFabl, vol. 3, $\mathrm{n}^{\mathrm{o}}$ 18, vers 6) une autre occurrence de enfin épistémique : Parolle qui n'est entendue, Sachiez enfin qu'ele est perdue («Sachez qu'en dernière analyse [glossaire : savoir en fin « être tout à fait sûr »], des paroles non comprises sont des paroles perdues »), à laquelle correspond, dans les manuscrits A (du $4^{\mathrm{e}}$ quart du $13^{\mathrm{e}}$ siècle) et $\mathrm{O}$ (du début du $14^{\mathrm{e}}$ siècle), sachiez de voir «sachez en vérité ». Or le manuscrit $\mathrm{H}$, de la fin du $13^{\mathrm{e}}$ siècle, présente Sachiés qu'ele est en fin perdue « sachez qu'à la fin, elle est perdue ».

13 Il ne nous paraît en effet pas indispensable de prévoir un item à part pour enfin récapitulatif en début d'énoncé.

14 Hansen, 2005b, 158, n. $6:$ dp. 1592 ; Hansen, 2005a : $48:$ dp. 1598. - La tradition est ininterrompue entre le $12^{\mathrm{e}}$ et le $16^{\mathrm{e}}$ siècle. Voici, à titre d'exemples, une attestation du $13^{\mathrm{e}}$, une du $14^{\mathrm{e}}$ et une du $15^{\mathrm{e}}$ siècle : Toute la plus fole [des femmes] est trop sage De pourquerre anui et damage, Et la plus sage trop voiseuse Et decevans et angoisseus.... Volages sont et poi estavles, Et sans mesure, enfin canjavles ( La plus sotte [des femmes] est encore assez intelligente pour chercher de l'ennui et du dommage, Et la plus sage est encore assez rusée et trompeuse et goulue... Elles sont volages et peu constantes, immodérées, bref, capricieuses ", déb. 13 ${ }^{\mathrm{e}}$ s., AmYdR, vers 3602); [dans une précédente lettre, la comtesse d'Artois a demandé quatre étoffes à son bailli : deux blanches, une rouge et une violette] nous vous prions [...] que, avec les escallates que nous vous avons mandé à pourveoir pour nous, vous nous en pourveez d'une bonne vermeille; enfin, nous envoierez-vous [...] deus escallates vermeilles [...], deus escallates blanches et une autre de droite coleur violete («nous vous prions [...] de nous envoyer, avec les étoffes déjà commandées, une bonne étoffe rouge ; en résumé, vous nous enverrez [...] deux étoffes rouges [...], deux blanches et une violette », 1335, Espinas \& Pirenne, $1920: 291$ = DMF2 s.v. bonté) ; [il est question de savoir si Bartolomeo Prihnano a été élu pape à juste titre] et après, tous ensemble... contredirent le fait, et fu escript et signé de leurs mains... ; enfin, concordablement en conclusion, fu de tous determiné que le dit Barthelmi n'estoit point pape, ainçois, par occupacion de tyranie tenoit le siege (« et ensuite tous contredirent ce fait, et ceci fut mis sur papier et signé par tous... ; en un mot, dans une déclaration unanime tous décidèrent que Bartolomeo n'était point pape, et qu'il avait obtenu le siège plutôt par un acte de tyrannie », 1404, ChrPisFaisS, vol. 2, p. 145).

15 Nous suivons en effet Bertrand \& Chanet (2005: 48) de même que Hansen (2005: 157) pour classer enfin5 énumératif parmi les pragmatèmes et non pas parmi les grammèmes, car le marqueur n'intervient pas au niveau référentiel, mais joue un rôle argumentatif (cf. le déjà ordinateur de Buchi, 2007b : 256). Paillard (2003 : 393), qui fonde sa distinction entre adverbe et mot du discours sur la notion de clôture discursive, analyse toutefois cet emploi comme non discursif.

16 À notre avis, la première partie du paragraphe I.C. (Avec lui j'appris vite l'hébreu, le sanscrit, et enfin le persan et l'arabe [« dans le temps du procès »]) représente la valeur de base I.A.

17 FEW : dp. ca 1150 (datation non utilisable dans notre contexte, car von Wartburg, qui reprend la définition de Gdf, mélange deux valeurs distinctes : «[adverbe qui sert à marquer que l'on conclut après une énumération ou 
ISBN 978-2-7598-0358-3, Paris, 2008, Institut de Linguistique Française

Diachronie, histoire de la langue

DOI $10.1051 / \mathrm{cmlf0} 161$

qu'une chose arrive après s'être fait attendre »]); Hansen, 2005a: $47:$ dp. 1587. Pour une attestation du $15^{\mathrm{e}}$ siècle (1452), cf. DMF2 s.v. tare (Comptes du roi René).

18 Les deux emplois distingués par Hansen (2005: 61) nous semblent relever en effet du même cas de figure.

19 TLF : dp. 1792.

20 Cadiot et al. (1985:227-228 [3.A.2.]) mettent à part un « enfin de connivence » (actualisé par exemple dans Untel $s$ 'est suicidé en prison, enfin tu vois ce que je veux dire), que nous rattachons au enfin performatif.

$21 \mathrm{TLF}: \mathrm{dp} .1902$.

22 TLF : dp. 1908

23 Cf. Nølke 2006, Smith 2006 et Waltereit 2006b. 